\title{
Large-Scale Screening of Asymptomatic Persons for SARS-CoV-2 Variants of Concern and Gamma Takeover, Brazil
}

\author{
Douglas Adamoski, ${ }^{1}$ Jaqueline Carvalho de Oliveira, Ana Claudia Bonatto, \\ Roseli Wassem, Meri Bordignon Nogueira, Sonia Mara Raboni, Edvaldo da Silva Trindade, \\ Emanuel Maltempi de Souza, SCB-UFPR COVID-19 team, ${ }^{2}$ Daniela Fiori Gradia ${ }^{1}$
}

We performed a large-scale severe acute respiratory syndrome coronavirus 2 screening campaign using 2 PCR-based approaches, coupled with variant genotyping, aiming to provide a safer environment for employees of Federal University in Curitiba, Brazil. We observed the rapid spread of the Gamma variant of concern, which replaced other variants in $<3$ months.

$\mathrm{O}$ ngoing screening for active severe acute respiratory syndrome coronavirus 2 (SARS-CoV-2) infections, coupled with contact tracing, can efficiently reduce viral transmission within the community (1). However, as new and more transmissible variants emerge, an increased number of cases can be observed across affected regions. This increase demands immediate action, such as consistent, uninterrupted genomic surveillance for regular evaluation of vaccination approaches (2), to curb virus spread.

Brazil has reaped the consequences of lack of genomic surveillance in a context of high seroprevalence. The variant of concern (VOC) Gamma (B.1.1.28/P.1) emerged in the city of Manaus, Brazil, at a time when three quarters of the population had tested positive for antibodies; it proceeded to spread across the city (3). The Gamma variant was able to replace its predecessor, the variant of interest (VOI) B.1.1.28/P.2, previously the dominant lineage in the region (4).

Author affiliations: Brazilian Biosciences National Laboratory, Brazilian Center for Research in Energy and Materials, Campinas, Brazil (D. Adamoski); Universidade Federal do Paraná, Curitiba, Brazil (D. Adamoski, J. Carvalho de Oliveira, A.C. Bonatto, R. Wassem, M.B. Nogueira, S.M. Raboni, E.S. Trindade, E.M. de Souza, D.F. Gradia)

DOI: https://doi.org/10.3201/eid2712.211326
Routine SARS-CoV-2 mass screening programs of asymptomatic persons and follow-up genotyping of samples are necessary measures to control the number of cases and prevent further infection surges $(5,6)$. Simplified approaches, such as multiplex quantitative PCR, provide a feasible, cost-effective way to discriminate samples and prioritize whole-genome sequencing efforts $(5,7)$. To provide a safer environment for the university community, we performed a large-scale screening for SARS-CoV-2 infections and VOCs in the Federal University of Paraná (Curitiba, Brazil) community. The study was approved by the University Research Ethics Committee (approval no. CAAE: 31687620.2.0000.0096).

\section{The Study}

During October 10, 2020-May 24, 2021, asymptomatic and mildly symptomatic persons within the community of the Federal University of Paraná were called for voluntary participation through social media and email. Eligible participants were members of the academic community (students, technicians, professors, or outsourced employees) or their relatives (grandparents, parents, siblings, or children) or household members. Saliva samples were selfcollected by using an individually wrapped plastic drinking straw, transferred to a prelabeled $2.0 \mathrm{~mL}$ microtube, and stored at $4^{\circ} \mathrm{C}$. Samples were transported to the laboratory in $<1 \mathrm{~h}$; total turnaround time to results was $<48 \mathrm{~h}$.

Samples were homogenized and allowed to settle for $30 \mathrm{~min}$ or centrifuged for $2 \mathrm{~min}(2,000 \times$ $g$ ). A quantity of $200 \mu \mathrm{L}$ from each specimen was

\footnotetext{
${ }^{1}$ These authors were co-principal investigators.

${ }^{2}$ Members of the group are listed at the end of this article.
} 
then pooled (8) in groups of 5 . We performed RNA extraction by using an automated magnetic EXTRACTA-RNA and DNA Viral kit (Loccus Biotecnologia, https://loccus.com.br). We performed amplification in 2 ways: on a QuantStudio5 instrument (Thermo Fisher Scientific, https://www.thermofisher.com) using AllPlex nCov-2019 reverse transcription PCR Master Mix Kit (SeeGene, https:// www.seegene.com) (1) or Molecular SARS-CoV-2 EDx (Bio-Manguinhos/FioCruz, https://www.bio. fiocruz.br) (2). If the pool rendered a positive result, we reprocessed samples individually.

We further evaluated positive samples by using 2 probe-based genotyping systems to detect VOCs. The first one was the Vogels et al. (7) multiplex approach to detect Spike $\Delta 69-70$ and Orf1a $\Delta 3675-3677$ deletions as an outcome for distinguishing Alpha, Beta, or Gamma and wild-type or other lineages (7). For this approach, we also included the Centers for Disease Control and Preventon N1 target and defined a cycle threshold $\left(C_{t}\right)$ of $<28$ on this particular target to evaluate the gene dropouts.

The second approach involved 3 allelic discrimination TaqMan assays (Thermo Fisher Scientific). The proposed readout was P.1 (K417T, N501Y, and E484K), P.2 (only E484K), B.1.1.7 (only N501Y), B.1.351 (N501Y and E484K, failure for K417T assay), and wild-type or others for the absence of mutated alleles (9). The discriminating power of this second assay made it possible to distinguish the B1.1.28/P.2 from the wild-type and the Beta/Gamma variants. We performed both assays by using GoTaq Probe 1-Step reverse transcription quantitative PCR System (Promega, https://www.promega.com) in the same instrument.

A total of 16 collection dates were recorded; 12,558 examinations were processed (Table) from the 7,249 persons who attended because some participants engaged in $>1$ day of collection. The number of attendees per collection date ranged from 162 to 1,737. The overall prevalence rate was $1.28 \%(161 / 12,558)$. Comparing these numbers to cases in the state of Paraná by the epidemiologic week of diagnosis (Figure 1, panel A), we found prevalence similar to the prevalence rate at the beginning of the state's second wave of SARS-CoV-2 infections (Figure 1, panel A).

We also evaluated all SARS-CoV-2-positive cases by using multiplex and singleplex genotyping approaches (Figure 2). From all 161 positive cases evaluated, the Vogels et al. (7) multiplex assay was invalidated in 46 (28.6\%) against 50 (31.1\%) in a Thermo Fisher 3-assay allelic detection approach because of the high $\mathrm{C}_{\mathrm{t}}$ values. Comparing the
Table. Collection dates, engagement, and positivity rates for severe acute respiratory syndrome coronavirus 2 infection, Curitiba, Brazil

\begin{tabular}{lcc}
\hline Date & Total tested & No. $(\%)$ positive \\
\hline 2020 Oct 2 & 275 & 0 \\
2020 Oct 19 & 279 & 0 \\
2020 Nov 6 & 510 & $6(1.18)$ \\
2020 Nov 24 & 1,265 & $34(2.69)$ \\
2020 Dec 8 & 1,070 & $17(1.59)$ \\
2021 Jan 12 & 1,692 & $23(1.36)$ \\
2021 Jan 26 & 1,737 & $14(0.81)$ \\
2021 Feb 9 & 1,615 & $16(0.99)$ \\
2021 Mar 29 & 196 & $1(0.51)$ \\
2021 Apr 12 & 157 & $4(2.55)$ \\
2021 Apr 20 & 872 & $2(0.23)$ \\
2021 Apr 26 & 162 & $4(2.47)$ \\
2021 May 4 & 884 & $12(1.36)$ \\
2021 May 10 & 177 & $1(0.56)$ \\
2021 May 18 & 1,431 & $20(1.4)$ \\
2021 May 24 & 236 & $7(2.97)$ \\
\hline Total & 12,558 & $161(1.28)$ \\
\hline
\end{tabular}

original $C_{t}$ value of detection, performance depreciated in samples with $C_{t}>30$ (Figure 2, panel B), as stated in the Thermo Fisher manual. Nevertheless, all genotyped cases were concordant between the 2 assays, considering that the Vogels et al. (7) assay alone does not discriminate between wild-type and B1.1.28/P.2 VOI.

Detection of the Gamma variant occurred on January 21, 2021, <2 weeks after the collapse of the healthcare system in Manaus. Prevalence of the Gamma variant was $9.1 \%$ on this date and increased to $42.9 \% 2$ weeks later (Figure 1, panel B). A possible explanation for this scenario is the increased transmission of this variant in $\geq 20 \%$ compared with the wild-type transmission rate (10), which is supported by the observation of a reproduction number of 1.5 in Paraná (http:// shiny.leg.ufpr.br/elias/covid19time) in the weeks before the death peak, the highest reproduction number observed during the pandemic. This increase in cases could be correlated with the subsequent collapse of the Curitiba city healthcare system and a surge of coronavirus disease deaths in the Paraná state, reaching values $>5 \%$ in the subsequent weeks. A similar scenario was also observed in Manaus, the origin of the Gamma variant; both SARS-CoV-2 cases and excess of burials in the city reached their highest levels during the pandemic to that point (4). Two factors could explain those observed surges: increased lethality of the Gamma variant - which is not yet defined (10) - and the actual collapse of healthcare systems, leading to poorer patient support. When testing activities resumed after the healthcare collapse, all cases became Gamma variant, completely displacing B1.1.28/P.2 VOI and wild-type cases in 3 months. 
Figure 1. COVID-19 diagnoses asymptomatic and variant frequency, and lethality, Paraná state, Brazil. A) COVID-19 diagnoses in Paraná and Federal University of Paraná (Curitiba, Brazil) mass testing program positivity rates by epidemiologic week. Blue bars summarize positive cases in Paraná by diagnosis day notified to state surveillance system through February 15, 2021. Red dots represent the fraction of positive cases in all samples from mass screening collection at Federal University of Paraná, smoothed by locally estimated scatterplot smoothing in the red line; pink shading indicates SE from locally estimated scatterplot smoothing fit. B) Overall lethality of COVID-19 in Paraná (purple line) and variant prevalence among asymptomatic and mildly symptomatic cases, by epidemiologic week. Numbers represent the absolute quantity of cases for each variant. Scales for the $y$-axes differ substantially to underscore patterns but do not permit direct comparisons. COVID-19, coro-
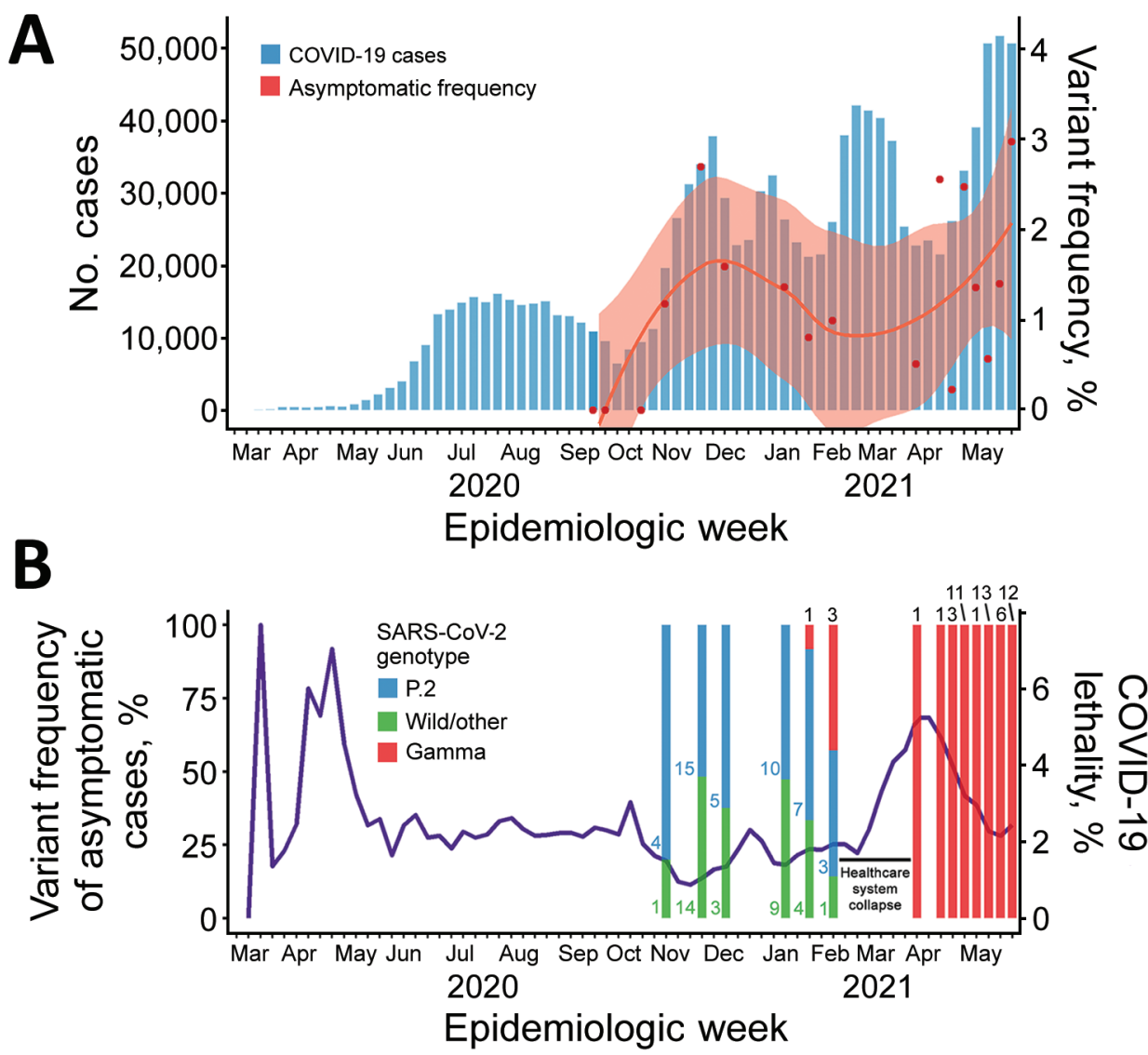

\section{Conclusions}

Analysis of saliva in pools as described in this study offers an inexpensive and easy-to-implement asymptomatic screening strategy. Thus, given the high rates of SARS-CoV-2 transmission, the risk for asymptomatic coronavirus disease spread and the importance of social distancing should continue to be stressed to the public until the vaccine is viable for large-scale application. Our mass testing pro- gram was intended to be accessible (every test was free-of-charge for the participant), reliable (all participants received their results and positive persons had a follow-up opportunity), and aimed to reach all social strata within the academic community (from professors to outsourced employees, which consisted mainly of socially, economically, and ethnically vulnerable groups), which are key characteristics of a strong mass testing system (11).

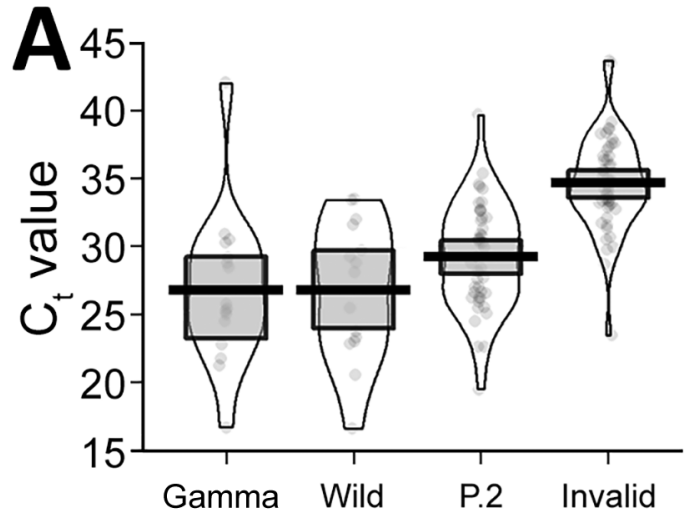

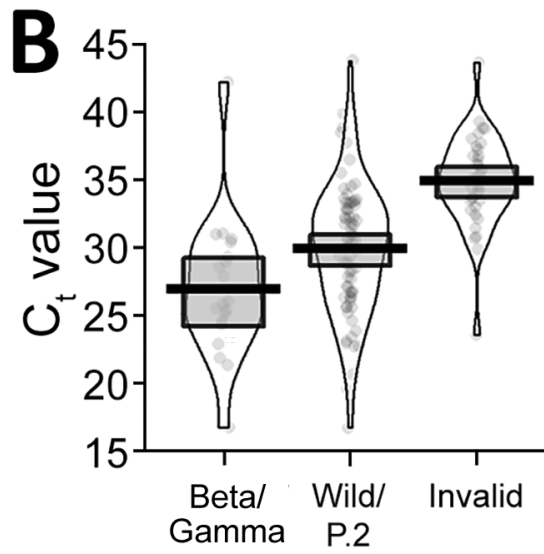

Figure 2. Violin plots of diagnostic $C_{t}$ values for severe acute respiratory syndrome coronavirus 2-positive cases in Paraná state, Brazil, and detection performance for singleplex (A) and multiplex (B) genotyping methods. Violin plots are made by kernelsmoothed density plots from the actual data (represented by the dots). Horizonal lines within boxes indicate medians; upper and lower box limits indicate interquartile ranges. $\mathrm{C}_{\mathrm{t}}$, cycle threshold. 
We found that both multiplex PCR and singleplex PCR approaches were valuable tools to evaluate the proportion of variants within genomic surveillance and were faster and less expensive than whole-genome sequencing approaches. Although those methods do not serve as substitutes for whole-genome sequencing, they could be an essential method to screen and select samples for further variant classification. Nevertheless, those approaches could demonstrate rapid spread of new variants and predict surges of SARS-CoV-2 infections, acting as a lighthouse for farreaching public health decisions.

This article was preprinted at https:/ /www.medrxiv.org/ content/10.1101/2021.06.18.21258649v1.

SCB-UFPR (Setor de Ciências Biológicas-Universidade Federal do Paraná) COVID-19 team members: Altina Bruna de Souza Barbosa, Beatriz Bocatte de Mattos, Bruna da Silva Soley, Carla Adriane Royer, Cibele Batina Rabelo, Cristina Kaehler, Diego Candido de Abreu, Guilherme Antonio Vendramin, Helyn Priscila de Oliveira Barddal, Letícia Dalla Vechia Henschel, Madson Silveira de Melo, Nathalie Carla Cardoso, and Rachel dos Santos de Sena de Vasconcelos.

\section{Acknowledgments}

We are grateful to Centro de Assessoria de Publicação Acadêmica (CAPA) from Federal University of Paraná for English revision. We are also grateful to Maria da Graça Bicalho for her support. We give special thanks to the team of volunteers; this job would not be possible without your help.

This work was supported by the PROPLAN/Federal University of Parana, Curitiba, Paraná, Brazil; FINEP-Funder of Studies and Projects, Ministry of Science, Technology and Innovation, Brazil, Institutional Network Project: Laboratories for Diagnostic tests for COVID-19 (0494/20).

\section{About the Author}

Dr. Adamoski is a substitute professor of genetics at the Curitiba Campus from Federal University of Paraná, now positioned as Researcher at National Laboratory of Biosciences. His primary research interests include mRNA binding proteins, splicing regulation, and genomics.

\section{References}

1. Mercer TR, Salit M. Testing at scale during the COVID-19 pandemic. Nat Rev Genet. 2021;22:415-26. https:/ / doi.org/ 10.1038/s41576-021-00360-w

2. Fontanet A, Autran B, Lina B, Kieny MP, Karim SSA, Sridhar D. SARS-CoV-2 variants and ending the COVID-19 pandemic. Lancet. 2021;397:952-4. https://doi.org/10.1016/ S0140-6736(21)00370-6

3. Buss LF, Prete CA Jr, Abrahim CMM, Mendrone A Jr, Salomon T, de Almeida-Neto C, et al. Three-quarters attack rate of SARS-CoV-2 in the Brazilian Amazon during a largely unmitigated epidemic. Science. 2021;371:288-92. https://doi.org/10.1126/science.abe9728

4. Faria NR, Mellan TA, Whittaker C, Claro IM, Candido DDS, Mishra S, et al. Genomics and epidemiology of the P.1 SARS-CoV-2 lineage in Manaus, Brazil. Science. 2021;372:815-21. https:// doi.org/10.1126/ science.abh2644

5. Matic N, Lowe CF, Ritchie G, Stefanovic A, Lawson T, Jang W, et al. Rapid detection of SARS-CoV-2 variants of concern, including B.1.1.28/P.1, British Columbia, Canada. Emerg Infect Dis. 2021;27:1673-6. https:// doi.org/10.3201/ eid2706.210532

6. Hamilton WL, Fieldman T, Jahun A, Warne B, Illingworth CJR, Jackson C, et al.; Cambridge COVID-19 group. Applying prospective genomic surveillance to support investigation of hospital-onset COVID-19. Lancet Infect Dis. 2021;21:916-7. https://doi.org/10.1016/ S1473-3099(21)00251-6

7. Vogels CBF, Breban MI, Ott IM, Alpert T, Petrone ME, Watkins AE, et al.; Brazil-UK CADDE Genomic Network; Network for Genomic Surveillance in South Africa. Multiplex qPCR discriminates variants of concern to enhance global surveillance of SARS-CoV-2. PLoS Biol. 2021;19:e3001236. https:// doi.org/10.1371/journal. pbio.3001236

8. Garg J, Singh V, Pandey P, Verma A, Sen M, Das A, et al. Evaluation of sample pooling for diagnosis of COVID-19 by real time-PCR: A resource-saving combat strategy. J Med Virol. 2021;93:1526-31. https://doi.org/10.1002/jmv.26475

9. Naveca FG, Nascimento V, de Souza VC, Corado AL, Nascimento F, Silva G, et al. COVID-19 in Amazonas, Brazil, was driven by the persistence of endemic lineages and P.1 emergence. Nat Med. 2021;27:1230-8. https://doi.org/10.1038/s41591-021-01378-7

10. Silva SJRD, Pena L. Collapse of the public health system and the emergence of new variants during the second wave of the COVID-19 pandemic in Brazil. One Health. 2021;13:100287. https://doi.org/10.1016/j.onehlt.2021.100287

11. Raffle AE, Pollock AM, Harding-Edgar L. Covid-19 mass testing programmes. BMJ. 2020;370:m3262. https://doi.org/10.1136/bmj.m3262

Address for correspondence: Douglas Adamoski, Brazilian Biosciences National Laboratory (LNBio), Brazilian Center for Research in Energy and Materials (CNPEM), Zip Code 13083-970, Campinas, Brazil; email: douglas.adamoski@gmail.com 